\title{
SISTEMA EXPERTO PARA LA PREVENCIÓN DE ENFERMEDADES BASADO EN EL CONSUMO DE ALIMENTOS COTIDIANOS
}

\section{EXPERT SYSTEM FOR ILLNESSES PREVENTION BASED ON THE DAILY FOOD CONSUMPTION}

\author{
Hugo Vega H., Augusto Cortez V. y Ana M. Huayna D. \\ FISI, Universidad Nacional Mayor de San Marcos, \\ Av. Germán Amezaga s/n Lima 1, Lima, Perú
}

\section{RESUMEN}

A lo largo de la existencia humana, el tratamiento de las enfermedades siempre ha sido una preocupación constante. Ahora que la población mundial se ha incrementado y las enfermedades se han multiplicado, la venta de medicamentos se ha convertido en uno de los principales negocios en el mundo.

Así mismo como se sabe, la medicina naturista es una alternativa muy importante en el tratamiento y prevención de las enfermedades. Teniendo en cuenta estas premisas, hemos realizado una importante investigación la misma que nos ha permitido generar el presente artículo.

El objetivo del presente artículo es explicar el modelamiento de un Sistema Experto para la Prevención y Tratamiento de Enfermedades basado en el consumo de potajes o dietas diarias

En resumen, el Sistema Experto (SE) que hemos modelado permite registrar como entrada síntomas, enfermedades o resultados de exámenes médicos de un paciente y genera como salida un listado de alternativas de platos o potajes que el paciente debe consumir, en su desayuno, almuerzo y cena. De ese modo, el paciente aliviará sus males, manteniendo en lo posible sus normales hábitos de consumo de alimentos. Por cierto el SE será el encargado de establecer el vínculo de los platos o potajes recomendados por el sistema con los productos naturales que alivian los síntomas registrados como entrada.

Obviamente, para cada paciente con síntomas diferentes, es de esperar que el SE le recomiende diferentes potajes.

Palabras clave: Sistema Experto, Base de Conocimiento, Prevención de Enfermedades, Medicina Natural.

\section{ABSTRACT}

Throughout human existence, the treatment of illnesses has always been a constant concern. Now that the world population has increased and the illnesses have multiplied, the sale of drugs has become one of the main businesses in the world.

Like we know, natural medicine is an important alternative in the treatment and prevention of illnesses. Given these assumptions, we have made an important investigation it has allowed us to generate this article.

The objective of this article is to explain the how to model an Expert System for the prevention and treatment of illnesses based on the daily food consumption.

In summary, the Expert System (ES) that we have modelling, permit you to register as entry symptoms, illnesses, or medical test results from a patient and generates as output a list of alternatives of foods that the patient should consume at breakfast, lunch, and supper. Thus, the patient relieves their symptoms, maintaining where possible their normal food consumption habits. Of course the ES will be responsible for the linkage of the dishes recommended by the system with natural products that relieve the symptoms registered as input.

Obviously, for each patient with different symptoms, it is hoped that ES recommend different dishes.

Keywords: Sistema Experto, Base de Conocimiento, Prevención de Enfermedades, Medicina Natural. 


\section{INTRODUCCIÓN}

Este trabajo presenta el diseño de un Sistema Experto que ofrece una alternativa para el tratamiento y la prevención de síntomas o enfermedades, en base a potajes, comidas normales, o dietas especiales.

Todo potaje está preparado por diversos productos, apoyados en la teoría científica se asignan las propiedades curativas a cada producto, y luego mediante una fórmula que se explica más adelante, se calcula el potencial curativo que tiene el potaje para aliviar diferentes síntomas o enfermedades.

EI SE toma como entrada los síntomas, enfermedades o resultados de exámenes médicos de un paciente, a través de su Base de Conocimientos detecta los productos naturales que poseen propiedades curativas para los síntomas ingresados y luego evalúa que potajes se preparan con dichos productos, luego evalúa que los potajes que son buenos para algún síntoma no sean perjudiciales o dañinos para otro síntoma o malestar del paciente de ser así los descarta y finalmente presenta todos los potajes que puede consumir en orden de prioridad o benéfico para mejorar la salud del paciente.

Lo más interesante de esta propuesta es que los pacientes podrán aliviar desde sus dolencias y malestares simples hasta realizar el tratamiento completo de ciertas enfermedades utilizando como medicamentos sus propios alimentos y sin hacer cambios drásticos en sus hábitos de consumo y sobre todo sin hacer gastos mayores en medicamentos.

\section{Definición de Sistema Experto (Se)}

Los Sistemas Expertos son sistemas que emulan el comportamiento de un experto humano para resolver un problema en un área de conocimiento específico y resuelve problemas utilizando una representación simbólica del conocimiento humano [1].

Un Sistema Experto es un sistema computarizado capaz de resolver problemas en el dominio en el cual posee conocimiento específico [2].

\section{Características de un sistema experto}

Las principales características de los Sistemas

Expertos son las siguientes [3] [6].

- Representan y utilizan conocimiento especializado de un determinado dominio

- Capacidad de razonar
- Muestra el mismo comportamiento del experto

- Representa el conocimiento simbólicamente

- Maneja problemas de dominio difícil

- Alto rendimiento en un dominio específico

- Examina y explica su propio razonamiento

\section{Arquitectura de un sistema experto}

Los principales componentes de un SE podrían resumirse en el siguiente esquema [5].

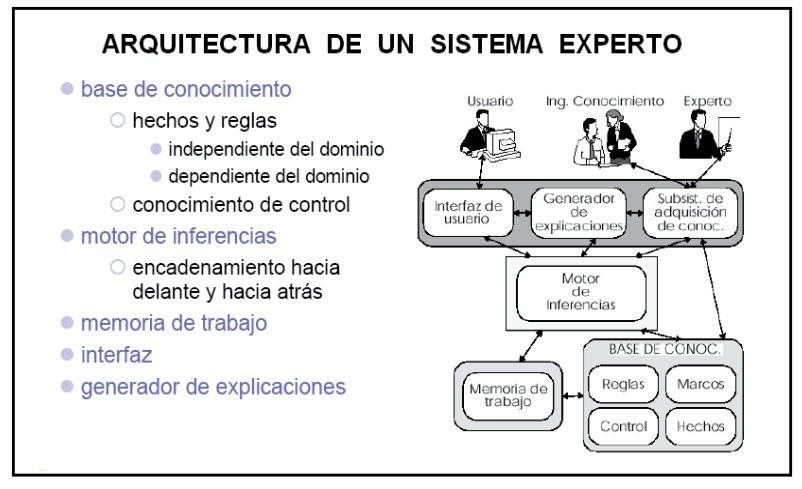

Figura 1. Arquitectura de un Sistema Experto

\section{a. Base de conocimiento}

Representa parte del universo donde se inserta el CONOCIMIENTO, el cual está conformado por todos los hechos y reglas del dominio de aplicación para la solución de un problema. Aquí es donde se representa toda la sapiencia y experiencia del experto humano [7].

\section{b. Motor de inferencias}

Viene a ser la unidad lógica con la que se extraen conclusiones de la base de conocimientos, una conclusión se produce mediante aplicación de las reglas sobre los hechos presentes [4]

Decide qué se debe aplicar, qué se debe hacer, resuelve conflictos nuevos, hace uso de la base de conocimiento.

Las funciones del mecanismo de inferencia son:

- Determinación de las acciones que tendrán lugar, el orden en que lo harán y cómo lo harán entre las diferentes partes del Sistema Experto.

- Determinar cómo y cuándo se procesarán las reglas, y dado el caso también la elección de qué reglas deberá procesarse.

- Control del diálogo con el usuario.

- Las formas de encadenamiento son:

- Encadenamiento hacia adelante (deductivo)

- Encadenamiento hacia atrás (inductivo). 


\section{c. Memoria de trabajo}

Contiene los hechos o conclusiones temporales, que se ha descubierto durante el análisis de un problema [4].

\section{d. Interfaz con el usuario}

Mediante este componente se realiza la interacción entre un sistema experto y un usuario. Es altamente interactiva y sigue el patrón de la conversación entre seres humanos. Para conducir este proceso de manera aceptable para el usuario es especialmente importante el diseño adecuado del interfaz de usuario. Esto puede requerir diseñar el interfaz usando menús o gráficos. Debe ser intuitivo, fácil en su manejo. No se debe olvidar que el SE simula al comportamiento de un experto. Debe ser cómodo y relativamente sencillo en cuanto al manejo ya que el usuario debe tener la máxima facilidad para manejar el software [4].

\section{e. Generador de explicaciones}

Una característica de los sistemas expertos es su habilidad para explicar su razonamiento. Usando el módulo del subsistema Generador de Explicaciones, un SE puede proporcionar una explicación al usuario de por qué está haciendo una pregunta y cómo ha llegado a una conclusión. Este módulo proporciona beneficios tanto al diseñador del sistema como al usuario. El diseñador puede usarlo para detectar errores y el usuario se beneficia de la transparencia del sistema [4].

\section{Lenguajes para desarrollar sistemas expertos}

Aunque en realidad se puede usar cualquier lenguaje para el desarrollo de SE existen algunos lenguajes creados exclusivamente para dicho propósito entre los cuales tenemos el LISP y el PROLOG. [5]

\section{a. LISP}

- El nombre LISP deriva de "LISts Processing" (Proceso de Listas). Las listas son una de las estructuras de datos importantes del LISP, y el código fuente del LISP en sí mismo está compuesto de listas.

- EI LISP fue inventado por John McCarthy en 1958 en el Instituto Tecnológico de Massachusetts (MIT). Fue publicado en 1960 en un paper de la ACM intitulado "funciones recursivas de expresiones simbólicas y su cómputo por la máquina, parte I."
- Desde ese entonces hasta el momento ha tenido importantes mejoras hasta convertirse en uno de los más importantes lenguajes de programación de Sistemas Expertos.

- El elemento fundamental en LISP es la lista, en el sentido más amplio del término, pues tanto los datos como los programas son listas. Las listas en LISP están delimitadas por paréntesis. Algunas de las funciones predefinidas de LISP tienen símbolos familiares (+ para la suma, * para el producto), pero otras como "car" y "cdr" sirven precisamente para descomponer las listas en sus componentes. "car" devuelve la cabeza de una lista y "cdr" su cola o resto.

- LISP fue uno de los primeros lenguajes de programación en incluir manejo de excepciones con las primitivas catch $y$ throw.

\section{Ejemplos}

Definición de funciones simples:

(defun vacia (I)

(cond ((null I) 1); si la lista esta vacía devuelve 1

(t 0); en otro caso devuelve 0))

(defun último (lista)

( cond ((null (cdr lista)) (car lista)) (t (ultimo (cdr lista)))))

Definición de funciones recursivas:

(defun factorial $(n)$

$$
\begin{aligned}
& (\text { if }(=0 \mathrm{n}) 1 \text {; caso base } \\
& \left.\left.\left({ }^{*} \mathrm{n}(\text { factorial }(-\mathrm{n} 1))\right) \text {; caso recursivo }\right)\right)
\end{aligned}
$$

Llamada a una función:

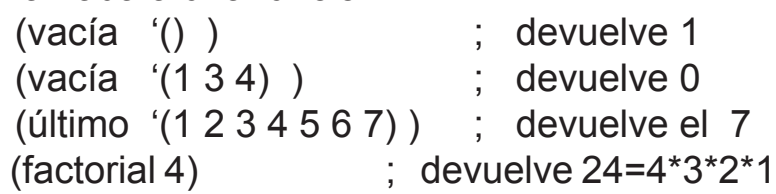

\section{b. PROLOG}

- El PROLOG, proveniente del francés PROgrammation en LOGique, es un lenguaje de programación lógica, es uno de los más importantes en el medio de investigación en Inteligencia Artificial.

- Fue ideado a principios de los años 70 en la Universidad de Aix-Marseille por los profesores Alain Colmerauer y Phillipe Roussel. Inicialmente se trataba de un lenguaje totalmente interpretado hasta que, a mediados de los 70, David H.D. Warren desarrolló un compilador capaz de traducir PROLOG en un conjunto de instrucciones de una máquina abstracta denominada Warren Abstract Machina ó WAM. 
- PROLOG se enmarca en el paradigma de los lenguajes lógicos, y se caracteriza por ser un lenguaje básicamente declarativo a diferencia de otros lenguajes más populares tales como el C, Java, etc.

- Los programas en PROLOG se componen de cláusulas de Horn, que son reglas del tipo "Si es verdad el antecedente, entonces es verdad el consecuente". No obstante, la forma de escribir las cláusulas de Horn es al contrario de lo habitual. Primero se escribe el consecuente y luego el antecedente. El antecedente puede ser una conjunción de condiciones que se denomina secuencia de objetivos. Cada objetivo se separa con una coma y puede considerarse similar a una instrucción o llamada a procedimiento de los lenguajes imperativos.

- Los Hechos, expresan relaciones entre objetos. Supongamos que queremos expresar el hecho de que "un carro tiene ruedas". Este hecho consta de dos objetos: "carro y "ruedas" y de una relación "tiene". La forma de representarlo en PROLOG es: tiene (carro, rueda).

\section{Ejemplos}

Si partimos de las siguientes premisas:

Todos los hombres son mortales $\rightarrow$ (Regla)

Sócrates es hombre

$\rightarrow$ (Hecho)

La conclusión lógica obvia es

Sócrates es mortal

$\rightarrow$ (Conclusión)

Este razonamiento lógico en PROLOG se programa de la siguiente manera:

$\operatorname{mortal}(\mathrm{X})$ :- hombre $(\mathrm{X}) . \rightarrow$ (Regla)

hombre(socrates). $\rightarrow$ (Hecho)

?- mortal(X). ). $\quad \rightarrow$ (Pregunta)

$\mathrm{X}=$ Sócrates; $\quad \rightarrow$ (Respuesta del Sistema)

\section{Metodología}

Planteamiento del Sistema Experto (SE)

El que presentamos permite la prevención de enfermedades en base al consumo adecuado de sus alimentos cotidianos (desayuno, almuerzo y cena).

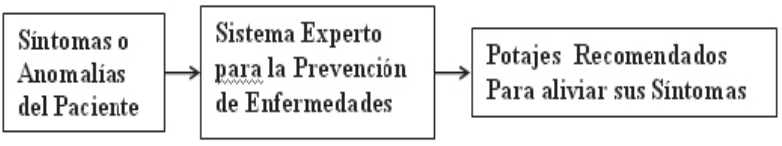

Figura 2. Esquema Básico del SE para la Prevención de Enfermedades.

\section{Base Conocimientos del SE}

La base de conocimientos (hechos y reglas) del SE contiene tres tópicos de conocimiento muy importantes que son los siguientes:

Los Síntomas por Paciente

El Potencial Curativo de los Productos por Síntomas

Ingredientes por Potaje

\section{a. Los síntomas por paciente:}

Los datos que requiere el SE son los malestares, dolencias, o síntomas de los pacientes, como dolor de cabeza, nauseas, escalofríos, fiebre, insomnio, pérdida de peso, pérdida de visión, etc. En la figura 3 representaremos este conocimiento indicando con uno (1) si un paciente sufre de un síntoma y con cero $(0)$ si en caso contrario.

\begin{tabular}{|l|c|c|c|c|c} 
& Sintoma 1 & Sintoma 2 & Sintoma 3 & & Sintoma 4 \\
\hline Paciente 1 & 1 & & 1 & & \\
\hline Paciente 2 & 1 & & & & 1 \\
\hline Paciente 3 & & 1 & 1 & & \\
\hline & & & & & \\
\hline Paciente 4 & & 1 & & &
\end{tabular}

Figura 3. Síntomas por paciente

De acuerdo a la figura 3 , la celda ( $p, s)$ toma el valor 1 cuando un Paciente $p$ TIENE el Síntoma s, en caso contrario será 0 es decir

$\operatorname{TIENE}(\mathrm{p}, \mathrm{s})=1$ Si el Paciente p tiene el Sintoma $\mathrm{s}$

$\operatorname{TIENE}(p, s)=0 \quad$ Si el Paciente p NO tiene el Sintoma $s$

\section{b. El potencial curativo de los productos por síntomas:}

En esta base de hechos se registra el potencial curativo o preventivo que poseen los productos naturales como frutas, verduras, tubérculos, cereales, y muchos otros productos que son, por lo general, ingredientes con los que se preparan los diversos potajes.

Si un producto cura o alivia un mal entonces diremos que posee un potencial curativo positivo (+1) y si un producto en lugar de curar o aliviar un mal, produce el efecto contrario, entonces diremos que posee un potencial curativo negativo $(-1)$ y si nos es ni bueno ni malo para un síntoma entonces su potencial será nulo (0). Esto lo podemos apreciar en la figura 4. 


\begin{tabular}{l|c|c|c|c|c} 
& Sintoma 1 & Sintoma 2 & Sintoma 3 & & Sintoma 4 \\
\hline Producto 1 & 1 & -1 & & & 1 \\
\hline Producto 2 & & 1 & & & -1 \\
\hline Producto 3 & 1 & 1 & 1 & & \\
\hline & & & & & \\
\hline Producto 4 & & 1 & 1 & &
\end{tabular}

Figura 4. Potencial curativo de los productos por síntomas.

De acuerdo a la figura 4 , la celda $(p, s)$ toma el valor 1 cuando un Producto $p$ CURA el Síntoma s. Si el Producto en lugar de curar es dañino para dicho Síntoma el valor será -1. Finalmente si el Producto $p$ no cura ni es dañino para el Síntoma s, entonces el valor será 0 .

$\operatorname{CURA}(p, s)=1$ Si el Producto $p$ cura el Síntoma s

CURA $(p, s)=0$ Si el Producto p NO cura el Síntoma s

CURA $(p, s)=-1$ Si el Producto p es dañino para el Síntoma s

\section{c. Ingredientes por potaje}

Aquí se registran los productos que son requeridos para el preparado de cada potaje; el valor que se registre debe ser el porcentaje que representa dicho producto en el potaje.

\begin{tabular}{l|c|c|c|c|c}
\hline & Potaje 1 & Potaje 2 & Potaje 3 & & Potaje 4 \\
\hline Producto 1 & 0,2 & 0,5 & 0,4 & & \\
\hline Producto 2 & 0,6 & & 0,2 & & 0,6 \\
\hline Producto 3 & 0,1 & 0,3 & & & \\
\hline & & & & & \\
\hline Producto 4 & & 0,1 & & & 0,1
\end{tabular}

Figura 5: Ingredientes por Potaje

De acuerdo a la figura 5, la celda (i, p) toma el valor del porcentaje en el que el Producto i interviene en el Potaje p. Si el Producto i no es usado para preparar el Potaje $p$ entonces el valor será 0 .

INGREDIENTE $(i, p)=$ Porcentaje del Producto $i$ en el Potaje $p$

INGREDIENTE $(i, p)=0$ Si elProducto $\mathrm{i}$ NO interviene en el Potaje $\mathrm{p}$

\section{d. Potajes recomendados para aliviar los síntomas:}

Considerando la Base de Conocimientos previamente especificada, el motor de inferencias del SE deduce el potencial curativo que posee cada potaje para el alivio de los diversos síntomas existentes.

\begin{tabular}{l|c|c|c|c|c} 
& Sintoma 1 & Sintoma 2 & Sintoma 3 & & Sintoma 4 \\
\hline Potaje 1 & $\mathbf{0 , 3}$ & $\mathbf{0 , 5}$ & $\mathbf{0 , 1}$ & & $\mathbf{0 , 4}$ \\
\hline Potaje 2 & $\mathbf{0 , 8}$ & $\mathbf{0 , 1}$ & $\mathbf{0 , 4}$ & & $\mathbf{0 , 5}$ \\
\hline Potaje 3 & $\mathbf{0 , 4}$ & $\mathbf{0 , 2}$ & $\mathbf{0}$ & & $\mathbf{0 , 2}$ \\
\hline & & & & & \\
\hline Potaje 4 & $\mathbf{0}$ & $\mathbf{0 , 7}$ & $\mathbf{0 , 1}$ & & $\mathbf{0 , 6}$
\end{tabular}

Figura 6. Potajes recomendados para aliviar sintomas

De acuerdo a la figura 6, el potencial curativo de un potaje $P$ respecto al síntoma $S$, especificado en la celda $(P, S)$ toma el valor de:

- La sumatoria de los potenciales curativos de los Productos que intervienen en el potaje $\mathrm{P}$ respecto al síntoma $S$ multiplicado por el porcentaje en el que dichos productos intervienen en el potaje P; siempre y cuando ninguno de los productos que intervengan en dicho potaje $P$ sean dañinos para el síntoma S.

- En el caso que uno o varios de los productos que intervengan en dicho potaje $P$ sean dañinos para el síntoma $S$, el valor de la celda $(P, S)$ estará dado por el valor más negativo de los diferentes valores que tomen la multiplicación del potencial curativo de los Productos que intervienen en el potaje $P$ respecto al síntoma $S$ multiplicado por el porcentaje en el que dichos productos intervienen en el potaje $\mathrm{P}$

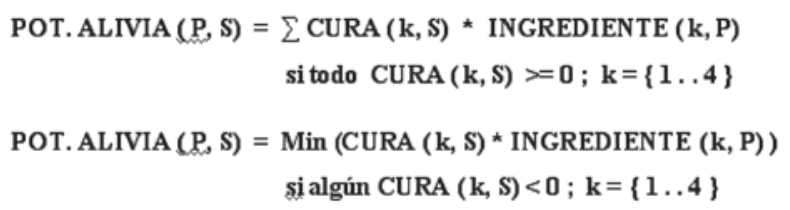

Funcionamiento del Sistema Experto (SE) a. Potajes recomendados para cada paciente:

Nuestro Sistema Experto, utilizando la base de conocimientos, mediante el motor de inferencias, calcula automáticamente la lista de potajes que debe consumir cada paciente para aliviar síntomas o malestares. Según los valores registrados en la base de conocimientos se llegaría a la siguiente conclusión.

\begin{tabular}{|l|c|c|c|c|c}
\hline & Potaje 1 & Potaje 2 & Potaje 3 & & Potaje 4 \\
\hline Paciente 1 & $\mathbf{0 , 4}$ & $\mathbf{1 , 2}$ & $\mathbf{0 , 4}$ & & $\mathbf{0 , 1}$ \\
\hline Paciente 2 & $-0,4$ & $\mathbf{1 , 3}$ & $\mathbf{0 , 6}$ & & 0,6 \\
\hline Paciente 3 & $\mathbf{0 , 6}$ & $-0,1$ & $-0,2$ & & $\mathbf{0 , 8}$ \\
\hline & & & & & \\
\hline Paciente 4 & $\mathbf{0 , 5}$ & $-0,1$ & $-0,2$ & & $\mathbf{0 , 7}$
\end{tabular}

Figura 7. Potajes recomendados para cada Paciente 
Para obtener los valores respectivos se han usado las siguientes fórmulas.

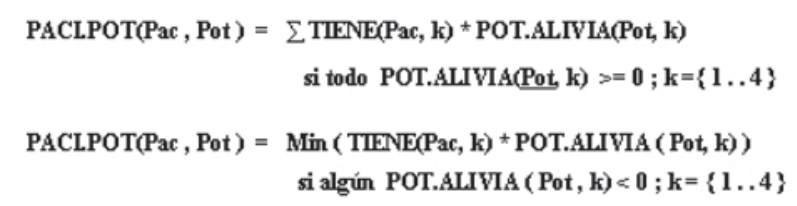

De acuerdo a la figura 7 , por ejemplo para el paciente 1 , el potaje mas recomendado seria el potaje 2 con una prioridad de 1.2, luego los potajes 1 y 3 con una prioridad de 0.4 y finalmente el potaje 4 con una prioridad de 0.1 .

\section{CONCLUSIONES}

Se vierte las principales conclusiones derivadas del estudio. Las Conclusiones constituyen el punto principal para futuras investigaciones, comprende la

Como resultado de este trabajo podemos concluir que los Sistemas Expertos son una gran alternativa para la solución de muchos problemas.

Con el presente sistema experto se podrá prevenir enfermedades ya que se permitirá el alivio de los síntomas a medida que se vayan presentando, evitando de esta manera que se incrementen los síntomas que posteriormente pueden causar graves enfermedades.

La debilidad que presenta este sistema experto es que no se ha considerado el problema de que las propiedades curativas de varios productos, al combinarse entre ellos, en lugar de incrementarse y ser más favorable a un síntoma, podrían causar el efecto contrario debido a las reacciones químicas de sus componentes.

La debilidad mencionada viene siendo tratada en un proyecto de investigación, para lo cual nos venimos apoyando en profesionales expertos en nutrición.

\section{AGRADECIMIENTOS}

Al Instituto de Investigaciones de la Facultad de Ingeniería de Sistemas e Informática de la UNMSM por su gran contribución en el desarrollo del presente artículo.

\section{REFERENCIAS}

[1] J.L.Alty "Sistemas Expertos, conceptos y ejemplos".Díaz de Santos - 1984

[2] J.P. Ignizio. "Introduction to expert systems". McGraw-Hill, 1991

[3] P. Lucas, L. van der Gaag. "Principles of expert systems". Addison-Wesley, 1991.

[4] P. Jackson. "Introduction to expert systems" Addison-Wesley, 1990.

[5] D. W. Rolston. "Principios de Int. Artificial y Sistemas Expertos". McGraw-Hill, 1990.

[6] Siemens Aktiengeseilschaft "Sistemas Expertos: Introducción a la técnica y aplicación" Marcombo -1988

[7] A. Gonzalez, "The engineering of knowledge-based systems". Prentice-Hall, 1993.

E-mail: hugovegahuerta@hotmail.com 